\title{
Lymph Node Surgery - Stepwise Retirement for the Breast Surgeon?
}

\author{
Julia Landin Walter P. Weber \\ Breast Center, University Hospital of Basel, Basel, Switzerland
}

\author{
Keywords \\ Breast cancer - Sentinel lymph node - Lymph node \\ dissection
}

\section{Summary}

Axillary lymph node dissection (ALND) has been standard of care for all patients with breast cancer until the 1990s. The stepwise retreat of breast surgeons from the axilla began after the introduction of the sentinel lymph node procedure. The evidence based clinical trend toward the omission of ALND has advanced to include patients with affected nodes, and several ongoing randomized controlled trials are evaluating the remaining indications for ALND. Conflicting with this trend toward less axillary surgery, indication and extent of regional nodal irradiation are currently broadened, equally supported by evidence from randomized trials. The present review summarizes this conflicting evidence, presents ongoing trials, and discusses the current and future optimal regional management of patients with affected nodes.

(c) 2016 S. Karger GmbH, Freiburg

\section{Evolution of Axillary Surgery}

Since the 1900s, axillary lymph node dissection (ALND) was standard of care for all patients with breast cancer. Many decades later, NSABP B04 was one of the first prospective studies to address axillary treatment. It randomized patients with clinically negative nodes into a group with total mastectomy and ALND - the current standard at the time - versus total mastectomy and regional irradiation versus total mastectomy alone without any axillary treatment [1]. Even though there was no difference in overall survival between the 3 groups, ALND remained standard of care for all patients, primarily for staging and regional control. Due to the morbidity associated with the procedure and the decreasing axillary node involvement over time, a series of randomized controlled trials (RCTs) have been initiated in the 1990s and early 2000s to question this paradigm in patients with clinically negative axillary lymph nodes. These trials can be divided into 4 categories: 1) omission of any surgical axillary staging in selected patients [25], 2) omission of axillary dissection in all patients with negative sentinel lymph nodes (SLN) [6-8], 3) omission of axillary dissection in selected patients with limited nodal disease in the SLN [9$11]$, and 4) axillary radiation versus axillary dissection [12, 13] or observation [14]. The trial findings supported the trend in clinical practice toward decreased rates of axillary dissection $[15,16]$ and established axillary radiation as valid alternative to dissection in selected patients.

\section{Residual Axillary Disease after Surgery}

The NSABP B04 trial showed that even in the absence of any surgical or adjuvant treatment, less than half of all axillary metastases progress to regional recurrence [1]. With the introduction of adjuvant radiation and systemic treatment, many more residual nodal metastases were treated and regional recurrence became rare. In 2010 for example, the NSABP B-32 study was published as the largest randomized surgical trial in breast cancer thus far [6]. In one arm, ALND was only performed when the sentinel node was positive. In the other arm, back-up ALND was performed in all patients and showed that the rate of false-negative sentinel nodes was $9.8 \%$. The trial demonstrated a rate of regional recurrence of $0.4 \%$ after ALND and only $0.7 \%$ without ALND despite the high false negative rate.

During the same randomization period from 1999 to 2004, patients with metastases in 1 or 2 sentinel nodes undergoing breast conserving surgery and adjuvant treatment were eligible for ACO-

\section{KARGER}

() 2016 S. Karger GmbH, Freiburg

Fax +497614520714 
Table 1. Ongoing clinical trials in axillary surgery

\begin{tabular}{|c|c|c|c|c|}
\hline Country/Name & Population & Randomization & Endpoint & Start \\
\hline $\begin{array}{l}\text { Italy } \\
\qquad \text { SOUND IEO S637/311 } \\
\text { NCT02167490 }\end{array}$ & \multicolumn{4}{|l|}{ US negative } \\
\hline $\begin{array}{l}\text { Germany } \\
\text { INSEMA } \\
\text { NCT02466737 }\end{array}$ & $\begin{array}{l}\text { cT1-2 cN0 } \\
\text { US negative }\end{array}$ & \multicolumn{3}{|l|}{ 2. $1-2 \mathrm{SLN}+\rightarrow$ ALND vs. no ALND } \\
\hline \multicolumn{5}{|l|}{$\begin{array}{l}\text { SERC/IPC 2012-001 } \\
\text { NCT01717131 }\end{array}$} \\
\hline $\begin{array}{l}\text { China } \\
\qquad \text { Z0011-China } \\
\text { NCT01796444 }\end{array}$ & \multicolumn{4}{|l|}{$1-2$ SLN+ } \\
\hline $\begin{array}{l}\text { Sweden } \\
\qquad \text { SENOMAC } \\
\text { NCT02240472 }\end{array}$ & $\begin{array}{l}\text { cT1-2 cN0 } \\
\text { cT1-2 iN1 } \\
1-2 \text { SLN+ }\end{array}$ & ALND vs. no ALND & BCSS & Jan 2015 \\
\hline $\begin{array}{l}\text { United Kingdom } \\
\text { POSNOC } \\
\text { NCT02401685 }\end{array}$ & $\begin{array}{l}\mathrm{cT} 1-2 \\
1-2 \mathrm{SLN}+\end{array}$ & $\begin{array}{l}\text { ALND or axillary radiotherapy vs. no } \\
\text { axillary treatment }\end{array}$ & $\begin{array}{l}\text { axillary } \\
\text { recurrence }\end{array}$ & Jan 2014 \\
\hline $\begin{array}{l}\text { Netherlands } \\
\text { BOOG 2013-07 } \\
\text { NCT02112682 }\end{array}$ & $\begin{array}{l}\text { cT1-2 cN0 } \\
1-3 \text { SLN+ } \\
\text { Mastectomy }\end{array}$ & $\begin{array}{l}\text { ALND or axillary radiotherapy vs. no } \\
\text { axillary treatment }\end{array}$ & $\mathrm{RRR}$ & June 2014 \\
\hline $\begin{array}{l}\text { USA } \\
\qquad \text { Alliance A011202 } \\
\text { NCT01901094 }\end{array}$ & $\begin{array}{l}\mathrm{cT} 1-3 \mathrm{cN} 1 \\
(\mathrm{~S}) \mathrm{LN}+\text { after NACT }\end{array}$ & $\begin{array}{l}\text { ALND + extended regional nodal } \\
\text { irradiation vs. axillary radiotherapy + } \\
\text { extended regional nodal irradiation }\end{array}$ & IBC-RFI & Feb 2014 \\
\hline $\begin{array}{l}\text { NCT }=\text { ClinicalTrials.gov ide } \\
\text { SLN = sentinel lymph node, } \\
\text { SLN }+=\text { SLN affected, iN1 = } \\
\text { (S)LN = lymph node (sentin } \\
\text { interval. }\end{array}$ & $\begin{array}{l}\mathrm{r}, \mathrm{cT} 1=\text { clinical stage } \\
=\text { distant disease-free } \\
\text { disease detected by im } \\
\text { on-sentinel), NACT }=\end{array}$ & $\begin{array}{l}\text { N0 = clinical stage N0 (no palpable lymp } \\
\text { ival, ALND = axillary lymph node disse } \\
\text { g, BCSS = breast cancer specific survival } \\
\text { adjuvant chemotherapy, IBC-RFI = inva }\end{array}$ & $\begin{array}{l}\text { des), US = ul } \\
\text { DFS = disea } \\
\mathrm{R}=\text { regional } \\
\text { breast cancer }\end{array}$ & $\begin{array}{l}\text { ind, } \\
\text { e survival, } \\
\text { ence rate, } \\
\text { rence-free }\end{array}$ \\
\hline
\end{tabular}

SOG Z0011 [10, 11]. They were randomized into one arm with ALND and one arm with no axillary-specific treatment. Despite the finding of residual nodal disease in $27 \%$ of patients treated by ALND in the control arm, the axillary recurrence rate was below $1 \%$ in the experimental arm without ALND. Taken together, these trials showed that most residual axillary lymph node metastases do not progress. The underlying reasons for this phenomenon most likely include regional control by host factors that are still poorly understood, and effective adjuvant radiation and systemic treatment.

\section{Conflicting Clinical Trends in Regional Management}

Conflicting with the trend toward less axillary surgery, radiation oncologists are broadening the indication for post-mastectomy irradiation in patients with positive nodes, based - among others on confirming recent data from the latest Lancet overview [17]. In this EBCTCG meta-analysis, postmastectomy radiotherapy has been shown to reduce breast cancer mortality for women with 1-3 affected axillary lymph nodes (risk ratio (RR) 0.80 ; $95 \%$ confidence interval (CI) $0.67-0.95$; log-rank $2, \mathrm{p}=0.01$ ). If given, post-mastectomy radiotherapy includes the chest wall and regional nodes in most patients [18]. Moreover, radiation oncologists are currently establishing the concept of extended regional lymph node irradia- tion based on evidence from 2 large phase III RCTs [19-21]. The majority of patients in MA.20 and more than half of the patients in EORTC 22922/10925 had positive axillary lymph nodes. Even though both trials showed improved loco-regional and distant control for extended radiation to the internal mammary and medial supraclavicular nodes, none of them achieved a significant overall survival benefit at 10 years. However, an improvement in overall survival of $3.7 \%$ at 8 years was shown for internal mammary nodal irradiation in a large population-based cohort study of patients with early-stage node-positive breast cancer (adjusted hazard ratio for death $0.82,95 \%$ CI $0.72-0.94 ; \mathrm{p}=0.005$ ) [22].

\section{Ongoing Randomized Controlled Trials}

Several surgical trials have been initiated to provide further evidence for the safety of omitting axillary surgery in selected clinically node-negative patients (table 1). Two of them investigate the omission of any surgical axillary staging, i.e. the omission of the SLN procedure, in patients with a negative preoperative ultrasound of the axilla. Until now, axillary ultrasound is used to identify axillary disease in clinically node-negative patients to omit the SLN procedure and proceed directly to axillary dissection. In the ACOSOG Z0011 era, this indication has become controversial inasmuch 
as patients with a positive preoperative axillary ultrasound may still undergo the SLN procedure without axillary dissection [23]. The results of the following 2 trials have the potential to establish a new indication for axillary ultrasound as a procedure to exclude highvolume axillary disease and spare patients any axillary surgery. The SOUND trial randomizes clinically node-negative patients with small breast cancers $(\leq 2 \mathrm{~cm})$ who are candidates for breast conserving surgery and irradiation into one group with the SLN procedure versus one group with no surgical axillary staging (observation arm). The SLN procedure is followed by axillary dissection in all patients with SLN macrometastases, which is perceived as overtreatment by many clinicians who apply the Z0011 protocol. The INSEMA trial has a similar first randomization, but includes patients with a clinical tumor size of up to $5 \mathrm{~cm}$ and randomizes patients with 1 or 2 positive SLN with macrometastases into one arm with ALND versus one arm without ALND. Therefore, the second randomization is designed to validate the findings of Z0011.

Several other ongoing RCTs primarily aim at validating the Z0011 protocol in different countries. Z0011-China strictly applies the Z0011 protocol to the same patient population in China. The other validation trials broadened the inclusion criteria compared with the original Z0011 protocol. The SERC/IPC 2012-001 trial in France, for example, randomizes all patients with positive SLN into one arm with ALND and one arm without ALND. The inclusion of patients with more than 2 positive SLN will add valuable evidence to clinical practice, since these patients were excluded from Z0011. A prospective study of a consecutive cohort of patients who met the Z0011 criteria at the time of initial surgery at the Memorial Sloan-Kettering Cancer Center showed that 29 of 287 patients (10.1\%) underwent completion ALND for $\geq 3$ positive SLNs [24]. On the other hand, the randomization of patients with isolated tumor cells or micrometastases into the ALND arm is nowadays considered overtreatment by many, at the latest since the publication of IBCSG 23-01 [9]. SENOMAC largely follows the Z0011 protocol in Sweden, but allows the inclusion of patients undergoing mastectomy and those with nodal disease detected by ultrasound. POSNOC investigates the Z0011 protocol in the United Kingdom, but allows axillary radiotherapy as alternative to ALND in the control arm. Similarly, BOOG 2013-07 from the Netherlands allows axillary radiotherapy or ALND to complete axillary treatment in the control arm, but includes patients with 1-3 positive SLN undergoing mastectomy. Notably, this trial allows randomization of mastectomy patients with SLN micrometastases into the axillary treatment arm, a patient population eligible for - but underrepresented in - IBCSG 23-01 [9].

The most progressive ongoing clinical trial of axillary management is Alliance A011202. It compares ALND with axillary radiation in patients with residual disease after chemotherapy, which was an exclusion criterion in both the AMAROS and the Z0011 trials. Eligibility criteria include initially clinically node-positive breast cancer that converted into clinically node-negative disease and at least 1 metastasis greater than $0.2 \mathrm{~mm}$ in sentinel or non-sentinel lymph nodes after chemotherapy. Patients in the ALND arm undergo extended regional nodal irradiation excluding the dissected axilla, while patients in the axillary radiation arm receive extended regional nodal irradiation including the full axilla. The trial tests the hypothesis that the AMAROS protocol in combination with extended regional nodal irradiation works in these patients without ALND inasmuch as chemotherapy-resistant lymph node metastases are as radiosensitive as chemotherapy-naive disease.

\section{Open Debates}

As in all clinical trials in oncology, patients are treated according to the standards that are in use when they are included. During follow-up, more effective treatment options may become available, potentially compromising the applicability of the findings to a contemporary patient population. The same is true for rigid in- and exclusion criteria, which are often necessary in terms of feasibility of the trial and later jeopardize its generalizability. Finally, methodological issues are frequent, and must be critically reviewed when determining the quality of the evidence. Many recent noninferiority trials were limited by lower-than-expected event rates resulting in a lack of statistical power. This is good for the patients in those studies, but problematic for trialists and, of course, clinicians trying to apply their results. Prominent examples are IBCSG 23-01, ACOSOG Z0011, and EORTC AMAROS [9-12]. Nevertheless, many of those landmark trials still managed to change clinical practice in selected pioneering centers of excellence, before their corroborative prospective surveillance data convinced critics that the protocols work and can be more widely adopted.

There is little controversy on the optimal management of sentinel node-negative patients and those with 4 or more positive nodes. However, for patients with 1-3 affected nodes, individualizing and optimizing treatment based on the available evidence is challenging. For example, the dramatic improvement in locoregional control for post-mastectomy and regional nodal irradiation and its impact on survival in patients with 1-3 positive nodes shown in the EBCTCG meta-analysis were based on a baseline 5 -year local-regional recurrence rate of $17 \%$, far in excess of what we see today. Generalized application of these data to today's patients disregards both relevant improvements in systemic therapy and the adverse effect of radiotherapy on complication rates after contemporary immediate breast reconstruction [25].

ACOSOG Z0011, EORTC-AMAROS, MA.20, and EORTC $22922 / 10925$ suggested fundamentally different approaches for similar patient populations [10-12, 20, 21]. ACOSOG Z0011 showed that many of these patients can be managed with no specific axillary treatment. EORTC-AMAROS validated axillary and medial supraclavicular radiation as equally effective and a potentially less harmful alternative to ALND in these patients. Finally, MA.20 and EORTC 22922/10925 suggested that some of these patients may benefit from extended regional nodal irradiation.

The main limitations of the MA.20 and EORTC 22922/10925 trials are that all patients underwent ALND and that many patients did not receive current systemic treatments, such as taxane chemotherapy, trastuzumab, and more effective endocrine strategies [26]. 
One limitation of ACOSOG Z0011 was the lack of standardization and detailed documentation of adjuvant radiation fields and the angles of the tangents, which makes it impossible to determine how much of the axilla was irradiated [27]. Since the no-axillary dissection arm in Z0011 was categorized as 'no further axillary treatment' and defined by 'no axillary dissection and no third-field nodal irradiation', this question became relevant. However, an attempt to reconstruct the radiation fields of Z0011 resulted in the receipt of only $30 \%$ of detailed radiotherapy records for centralized review [28]. Critics of ACOSOG Z0011 felt reassured by this publication inasmuch as a substantial amount of patients received directed regional nodal radiotherapy using $\geq 3$ fields. However, the facts that most patients treated in Z0011 received tangential radiotherapy alone and some did not receive radiotherapy at all make it very unlikely that the very low regional recurrence rate of $0.9 \%$ (4/436) in the SLN alone arm at a median follow-up of 6.3 years was observed due to protocol-prohibited nodal fields. Another limitation of Z0011 was the limited duration of follow-up, a self-resolving issue since 10-year follow-up results are expected soon. Finally, particularly in Europa, there is still no uniform consensus on the application of the Z0011 data to several subgroups, such as young patients with estrogen receptor-negative or high-grade breast cancer, due to a relative underrepresentation of these patients in the trial [29]. Corroborative prospective surveillance data from centers that adopted the Z0011 protocol more than 5 years ago and pending results from various Z0011 validation trials will close that debate in the near future.

\section{Current and Future Axillary Treatment}

To date, most patients with invasive breast cancer should undergo the SLN procedure; however, SOUND and INSEMA may challenge this paradigm within the next decade or two. Patients with micrometastases or isolated tumor cells can forego ALND [9-11]. Most patients with macrometastases in 1 or 2 sentinel nodes undergoing breast conserving surgery, adjuvant wholebreast irradiation, and systemic treatment should not undergo ALND $[10,11,30]$. They can be treated according to the Z0011 protocol without axilla-specific treatment or according to EORTC AMAROS with axillary radiotherapy; the optimal patient selection for one or the other remains a subject for further study. In the absence of a trial of extended regional nodal irradiation following the sentinel node procedure alone, individualized treatment decisions on the extent of irradiation should be based on the patient's baseline risk of recurrence.

The remaining indications for ALND include confirmed nodepositive breast cancer with a large primary $(>5 \mathrm{~cm})$ or high-volume nodal disease ( $\geq 3$ positive SLN, gross extranodal disease, palpable lymph node metastases), residual nodal disease after neoadjuvant chemotherapy, and patients with at least one sentinel node macrometastasis undergoing mastectomy. The latter, however, is only valid if the number of positive nodes removed by ALND is needed to evaluate the indication for post-mastectomy radiotherapy. If post-mastectomy radiotherapy including the regional nodes is indicated according to local protocols due to the positive SLN per se, ALND can be safely omitted, a lesson learned from EORTC AMAROS [12, 31]. Finally, potential predictors of high nodal disease volume are sometimes used in clinical practice to indicate ALND; the most common are lymph node metastases detected by imaging before surgery and microscopic extranodal disease in the SLN [3234]. While the extent of extracapsular extension may influence the need for ALND [35], a positive imaging-guided axillary lymph node needle biopsy does not accurately predict the need for ALND [23]. BOOG 2013-07 from the Netherlands, SERC/IPC 2012-001 from France and the Alliance trial A011202 may eliminate most of the remaining indications for ALND, potentially leaving only confirmed palpable lymph node metastases as the last absolute indication for ALND in the near future. With decreasing use of ALND, opportunities for junior staff, fellows, and residents to practice this procedure will continue to decline. In parallel, the technical complexity of performing ALND will continue to increase in the more advanced, chemotherapy-resistant, or recurrent disease. Therefore, breast surgeons will not retire from lymph node surgery; instead, they will be challenged by performing more difficult procedures with less experience.

\section{Disclosure Statement}

The authors received no financial support for this work and have no conflicts of interest.

\section{References}

1 Fisher B, Jeong JH, Anderson S, et al.: Twenty-five-year follow-up of a randomized trial comparing radical mastectomy, total mastectomy, and total mastectomy followed by irradiation. N Engl J Med 2002;347:567-575.

2 Agresti R, Martelli G, Sandri M, et al.: Axillary lymph node dissection versus no dissection in patients with T1N0 breast cancer: a randomized clinical trial (INT09/98). Cancer 2014;120:885-893.

3 Martelli G, Boracchi P, De PM, et al.: A randomized trial comparing axillary dissection to no axillary dissection in older patients with T1N0 breast cancer: results after 5 years of follow-up. Ann Surg 2005;242:1-6.
4 Martelli G, Boracchi P, Ardoino I, et al.: Axillary dissection versus no axillary dissection in older patients with T1N0 breast cancer: 15-year results of a randomized controlled trial. Ann Surg 2012;256:920-924.

5 Rudenstam CM, Zahrieh D, Forbes JF, et al.: Randomized trial comparing axillary clearance versus no axillary clearance in older patients with breast cancer: first results of International Breast Cancer Study Group Trial 10-93. J Clin Oncol 2006;24:337-344.
6 Krag DN, Anderson SJ, Julian TB, et al.: Sentinellymph-node resection compared with conventional axillary-lymph-node dissection in clinically node-negative patients with breast cancer: overall survival findings from the NSABP B-32 randomised phase 3 trial. Lancet Oncol 2010;11:927-933.

7 Mansel RE, Fallowfield L, Kissin M, et al.: Randomized multicenter trial of sentinel node biopsy versus standard axillary treatment in operable breast cancer: the ALMANAC Trial. J Natl Cancer Inst 2006;98:599-609. 
8 Veronesi U, Paganelli G, Viale G, et al.: A randomized comparison of sentinel-node biopsy with routine axillary dissection in breast cancer. N Engl J Med 2003; 349:546-553.

9 Galimberti V, Cole BF, Zurrida S, et al.: Axillary dissection versus no axillary dissection in patients with sentinel-node micrometastases (IBCSG 23-01): a phase 3 randomised controlled trial. Lancet Oncol 2013;14:297-305.

10 Giuliano AE, McCall L, Beitsch P, et al.: Locoregional recurrence after sentinel lymph node dissection with or without axillary dissection in patients with sentinel lymph node metastases: the American College of Surgeons Oncology Group Z0011 randomized trial. Ann Surg 2010;252:426-432.

11 Giuliano AE, Hunt KK, Ballman KV, et al.: Axillary dissection vs no axillary dissection in women with invasive breast cancer and sentinel node metastasis: a randomized clinical trial. JAMA 2011;305:569-575.

12 Donker M, van TG, Straver ME, et al.: Radiotherapy or surgery of the axilla after a positive sentinel node in breast cancer (EORTC 10981-22023 AMAROS): a randomised, multicentre, open-label, phase 3 non-inferiority trial. Lancet Oncol 2014;15:1303-1310.

13 Louis-Sylvestre C, Clough K, Asselain B, et al.: Axillary treatment in conservative management of operable breast cancer: dissection or radiotherapy? Results of a randomized study with 15 years of follow-up. J Clin Oncol 2004;22:97-101.

14 Veronesi U, Orecchia R, Zurrida S, et al.: Avoiding axillary dissection in breast cancer surgery: a randomized trial to assess the role of axillary radiotherapy. Ann Oncol 2005; 16:383-388.

15 Bilimoria KY, Bentrem DJ, Hansen NM, et al.: Comparison of sentinel lymph node biopsy alone and completion axillary lymph node dissection for node-positive breast cancer. J Clin Oncol 2009;27:2946-2953.

16 Weber WP, Barry M, Stempel MM, et al.: A 10-year trend analysis of sentinel lymph node frozen section and completion axillary dissection for breast cancer: are these procedures becoming obsolete? Ann Surg Oncol 2012;19:225-232.
McGale P, Taylor C, Correa C, et al.: Effect of radiotherapy after mastectomy and axillary surgery on 10-year recurrence and 20-year breast cancer mortality: metaanalysis of individual patient data for 8135 women in 22 randomised trials. Lancet 2014;383:2127-2135.

18 Coates AS, Winer EP, Goldhirsch A, et al.: Tailoring therapies - improving the management of early breast cancer: St Gallen International Expert Consensus on the Primary Therapy of Early Breast Cancer 2015. Ann Oncol 2015;26:1533-1546.

19 Budach W, Kammers K, Boelke E, Matuschek C: Adjuvant radiotherapy of regional lymph nodes in breast cancer - a meta-analysis of randomized trials. Radiat Oncol 2013;8:267.

20 Poortmans PM, Collette S, Kirkove C, et al.: Internal mammary and medial supraclavicular irradiation in breast cancer. N Engl J Med 2015;373:317-327.

21 Whelan TJ, Olivotto IA, Parulekar WR, et al.: Regional Nodal Irradiation in Early-Stage Breast Cancer. N Engl J Med 2015;373:307-316.

22 Thorsen LB, Offersen BV, Dano H, et al.: DBCG-IMN: A population-based cohort study on the effect of internal mammary node irradiation in early node-positive breast cancer. J Clin Oncol 2016;34:314-320.

23 Pilewskie M, Mautner SK, Stempel M, et al.: Does a positive axillary lymph node needle biopsy result predict the need for an axillary lymph node dissection in clinically node-negative breast cancer patients in the ACOSOG Z0011 era? Ann Surg Oncol 2016;23:11231128.

24 Dengel LT, Van Zee KJ, King TA, et al.: Axillary dissection can be avoided in the majority of clinically node-negative patients undergoing breast-conserving therapy. Ann Surg Oncol 2014;21:22-27.

25 Jagsi R, Jiang J, Momoh AO, et al.: Complications after mastectomy and immediate breast reconstruction for breast cancer: a claims-based analysis. Ann Surg 2016; 263:219-227.

26 Burstein HJ, Morrow M: Nodal irradiation after breast-cancer surgery in the era of effective adjuvant therapy. N Engl J Med 2015;373:379-381.
27 Giuliano AE: Reply to Letter: Are the standard tangential breast irradiation fields used in the ACOSOG Z0011 trial really covering the entire axilla? Ann Surg 2013;257:e2.

28 Jagsi R, Chadha M, Moni J, et al.: Radiation field design in the ACOSOG Z0011 (Alliance) trial. J Clin Oncol 2014;32:3600-3606.

29 Hoffmann J, Souchon R, Lebeau A, et al.: German, Austrian and Swiss consensus conference on the diagnosis and local treatment of the axilla in breast cancer. Eur J Cancer 2013;49:2277-2283.

30 Lyman GH, Temin S, Edge SB, et al.: Sentinel lymph node biopsy for patients with early-stage breast cancer: American Society of Clinical Oncology clinical practice guideline update. J Clin Oncol 2014;32:1365-1383.

31 Morrow M: It is not always necessary to do axillary dissection for T1 and T2 breast cancer - point. Cancer Res 2013;73:7151-7154.

32 Cools-Lartigue J, Sinclair A, Trabulsi N, et al.: Preoperative axillary ultrasound and fine-needle aspiration biopsy in the diagnosis of axillary metastases in patients with breast cancer: predictors of accuracy and future implications. Ann Surg Oncol 2013;20:819-827.

33 Moorman AM, Bourez RL, Heijmans HJ, Kouwenhoven EA: Axillary Ultrasonography in Breast Cancer Patients Helps in Identifying Patients Preoperatively with Limited Disease of the Axilla. Ann Surg Oncol 2014;21:2904-2910.

34 van Wely BJ, de Wilt JH, Schout PJ, et al.: Ultrasoundguided fine-needle aspiration of suspicious nodes in breast cancer patients; selecting patients with extensive nodal involvement. Breast Cancer Res Treat 2013;140: 113-118.

35 Gooch J, King Ta, Eaton A, et al.: the extent of extracapsular extension may influence the need for axillary lymph node dissection in patients with T1-T2 breast cancer. Ann Surg Oncol 2014. 